\title{
Physical Model for Plaque Action in the Tooth-Plaque-Saliva System
}

\author{
WILLIAM I. HIGUCHI, FUDAH YOUNG, JESUS L. LASTRA, and THEODORE \\ KOULOURIDES \\ College of Pharmacy, University of Michigan, Ann Arbor, Michigan 48104 and School \\ of Dentistry, University of Alabama Medical Center, Birmingham, Alabama 35233
}

A physical model describing the interrelationships of demineralization, remineralization, plaque thickness, glucose levels, and plaque enzymatic activity was presented. Selection of constants and variations of the parameters were kept in the range of possible in vivo situations. The results of calculations were discussed and correlated with the results of in vivo studies.

Recent work has demonstrated that the physical model approach should greatly aid in understanding enamel demineralization, remineralization, and enamel reactions in general. Such research has already led to a systematic, self-consistent, mechanistic description of the acid demineralization kinetics for dental enamel. ${ }^{1-5}$ The roles played by the mineral phase, the type and concentration of acid buffers, $\mathrm{pH}$, and solution of fluoride have been investigated.

The physical model approach is not intended to be "theory" in the usual sense. The purpose of the models are not generally to determine whether they are correct or incorrect on an all-or-none basis. Instead, such models most often provide, in the light of experimental data, the initial quantitative inter-relationships that considered intuition alone may have been unable to provide. From these models, suitable in vitro, in situ, or in vivo experiments are designed; from these experiments, refinements of the models may follow.

Evidence ${ }^{6,7}$ involving plaque, oral bacteria, carbohydrate substrate, and enamel

This investigation was supported in part by USPHS Research Grant DE-01830 from the National institute of Dental Research, National Institutes of Health, Bethesda, Md.

Received for publication July 22, 1968. demineralization supported, directly or indirectly, the concept that acid production in cariogenic plaque is the decisive factor causing dental caries. In some instances reasonably self-consistent correlations have been shown, but in other instances little or no correlation has been observed. Because of the lack of studies involving the general interrelationships among the variables involved in laboratory tests, there is an increasing need to correlate and interpret the many variables of dental caries at a molecular level.

Clinically, the most easily identifiable lesion of dental caries is the formation of a cavity in the tooth. Although numerous factors influence the course of the disease, the incipient caries is primarily a lesion of the dental enamel. The necessary conditions for dental caries can be generally considered in terms of contributing factors in the host, the oral bacteria, and the dietary substrates. The sufficient condition is the dissolution of dental enamel in an acid environment, which is created by the microbial metabolism of the carbohydrate substrates in the plaque.

Because of the success achieved by the use of physical models in describing the general behavior of tooth minerals in acidic buffers, ${ }^{1-5}$ it was decided to explore this approach in connection with the mechanism of the action of plaque on dental enamel. In the present report this method is generalized to include most aspects of dental caries and calculus formation, especially the basic interrelationships among the mineral phase, plaque, and saliva variables. Mathematical relations involving simultaneous diffusion and chemical reactions of species existing in the enamel-plaque-saliva 
system $^{8,9}$ have been presented wherever possible.

\section{Materials and Methods}

Formulation OF a STEADY-STATE MODEL.-The basic model for demineralization and remineralization is presented in Figures 1 and 2. Enamel was assumed to be hydroxyapatite, which is the thermodynamically governing phase. In the saliva, the ions and molecules considered ware $\mathrm{Ca}^{2+}, \mathrm{H}_{2} \mathrm{PO}_{4}^{-}, \mathrm{HPO}_{4}^{-}, \mathrm{HCO}_{3}^{-}, \mathrm{H}_{2} \mathrm{CO}_{3}$, $\mathrm{H}^{+}$, and the carbohydrate substrate. It was assumed that the diffusion of these species, the bacteria-produced acid molecules, and their anions take place in the plaque, which is constantly bathed in the saliva. Phosphates and carbonates were considered to be neither generated nor used in the plaque.

Glucose (S) was assumed to be the only carbohydrate substrate responsible for acid production, and for the sake of simplicity, lactic acid was considered to be the principal acid product. ${ }^{10,11}$ The kinetic behavior of the glycolysis reaction between glucose

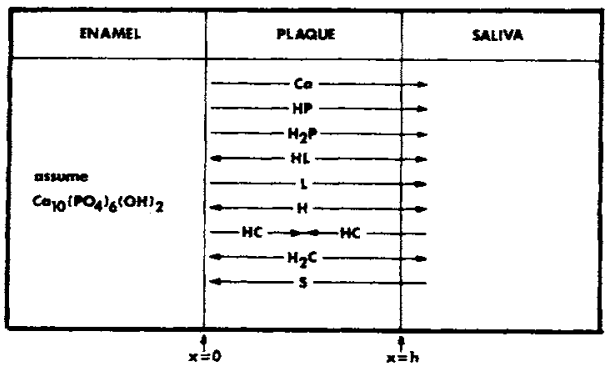

Frg 1.-Diagram for demineralization in an enamel-plaque-saliva system. It is assumed that the mineral phase is at equilibrium with the ions and molecules involved at the enamelplaque interface, ie, at $x=0$.

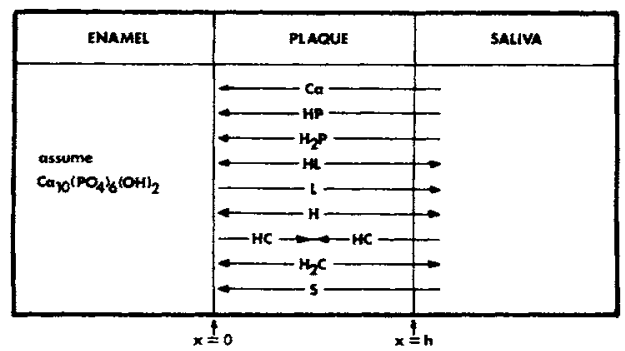

FI 2.-Diagram for remineralization in an enamel-plaque-saliva system. and plaque material was assumed to follow the Michaelis-Menten type of rate law in which the rate varies linearly with substrate concentration at low concentrations (first order kinetics), and becomes independent of substrate concentration (zero order kinetics) at high concentrations. ${ }^{22}$ The $\mathrm{Mi}$ chaelis constants, $k_{1}$ and $k_{2}{ }^{*}$, served as a representation of the apparent enzymatic activities of the plaque or bacteria in acid production.

For a one dimensional problem (Fig 1, 2) with the axis, $x$, perpendicular to the enamel-plaque interface, the following steady-state equations can be written to describe the simultaneous diffusion and chemical reactions of the species in the plaque per unit area:

$$
\begin{gathered}
D_{\mathrm{Ca}} \frac{d^{2}[\mathrm{Ca}]}{d x^{2}}=0 \\
D_{\mathrm{NP}} \frac{d^{2}[\mathrm{HP}]}{d x^{2}}-\phi_{1}-\phi_{2}-\phi_{4}=0 \\
D_{\mathrm{H}_{2} \mathrm{P}} \frac{d^{2}\left[\mathrm{H}_{2} \mathrm{P}\right]}{d x^{2}}+\phi_{1}+\phi_{2}+\phi_{4}=0 \\
D_{\mathrm{HL}} \frac{d^{2}[\mathrm{HL}]}{d x^{2}}-\phi_{2}+\phi_{3}+R=0 \\
D_{\mathrm{L}} \frac{d^{2}[\mathrm{~L}]}{d x^{2}}+\phi_{2}-\phi_{3}=0 \\
D_{\mathrm{H}_{2} \mathrm{C}} \frac{d^{2}\left[\mathrm{H}_{2} \mathrm{C}\right]}{d x^{2}}-\phi_{4}-\phi_{5}=0 \\
D_{\mathrm{HC}} \frac{d^{2}[\mathrm{HC}]}{d x^{2}}+\phi_{4}-\phi_{5}=0 \\
D_{\mathrm{H}} \frac{d^{2}[\mathrm{H}]}{d x^{2}}-\phi_{1}-\phi_{3}-\phi_{5}=0 \\
D_{\mathrm{S}} \frac{d^{2}[\mathrm{~S}]}{d x^{2}}-R=0
\end{gathered}
$$

These expressions for Fick's law of diffusion take into account the mass balances of the chemical reactions of the various species involved. ${ }^{13}$ The $D$ 's in the equations (1-9) are the respective diffusion coefficients for $\mathrm{Ca}^{2+}, \mathrm{HPO}=, \mathrm{H}_{2} \mathrm{PO}_{4}^{-}, \mathrm{H}_{2} \mathrm{CO}_{3} \mathrm{HCO}_{3}^{-}$, $\mathrm{H}^{+}$, glucose (S), lactic acid (HL), and its anion (L). Quantities in the brackets are terms expressing the concentrations of the respective species. The $\phi$ 's and $R$ are the

* The parameters, $k_{1}$ and $k_{2}$, represent the apparent overall rate constants applicable in the plaque milieu. 
rates of reaction per unit volume for the following reactions:

$$
\begin{array}{ll}
\mathrm{HP}+\mathrm{H} \rightarrow \mathrm{H}_{2} \mathrm{P} & \phi_{1} \\
\mathrm{HP}+\mathrm{HL} \rightarrow \mathrm{H}_{2} \mathrm{P}+\mathrm{L} & \phi_{2} \\
\mathrm{H}+\mathrm{L} \rightarrow \mathrm{HL} & \phi_{3} \\
\mathrm{HP}+\mathrm{H}_{2} \mathrm{C} \rightarrow \mathrm{H}_{2} \mathrm{P}+\mathrm{HC} & \phi_{4} \\
\mathrm{H}+\mathrm{HC} \rightarrow \mathrm{H}_{2} \mathrm{C} & \phi_{5} \\
\mathrm{~S}+\mathrm{E} \rightarrow \mathrm{HL} & R
\end{array}
$$

$R$ is the rate of lactic acid production and $E$ represents the effective enzyme systems in the bacteria. $R$ is a function of glucose concentration (S) and assumed to follow the Michaelis-Menten rate law, ie,

$$
R=\frac{k_{1}(\mathrm{~S})}{1+k_{2}(\mathrm{~S})}
$$

where $k_{1}$ and $k_{2}$ are rate constants. Along with the equations (1-10), the following equilibrium expressions apply at the enamelplaque interface and at any positions in the plaque, and in the saliva, ie, at $x \geqslant 0$ :

$$
\begin{aligned}
& K_{2 \mathrm{P}}=\frac{(\mathrm{H})(\mathrm{HP})}{\left(\mathrm{H}_{2} \mathrm{P}\right)} \\
& K_{\mathrm{BP}}=\frac{(\mathrm{H})(\mathrm{P})}{(\mathrm{HP})} \\
& K_{\mathrm{HL}}=\frac{(\mathrm{H})(\mathrm{L})}{(\mathrm{HL})} \\
& K_{\mathrm{H}_{2} \mathrm{C}}=\frac{(\mathrm{H})(\mathrm{HC})}{\left(\mathrm{H}_{2} \mathrm{C}\right)} \\
& K_{W}=(\mathrm{H})(\mathrm{OH})
\end{aligned}
$$

where $K_{W}$ is the ion product for water. At the enamel-plaque interface, hydroxyapatite governs the equilibrium conditions, ie, at $x=0$

$$
K_{\mathrm{HAP}}=(\mathrm{Ca})_{0}^{10}(\mathrm{P})_{0}^{6}(\mathrm{OH})_{0}^{2}
$$

where $K_{\mathrm{HAP}}$ is the solubility product of hydroxyapatite. Considering the enamelplaque interface, $x=0$, and the plaquesaliva interface, $x=h$, where $h$ is the plaque thickness, there are other boundary conditions which can be established in the region $0=x=h$ :

$$
\begin{gathered}
D_{\mathrm{H}_{2} \mathrm{O}} \frac{d\left(\mathrm{H}_{2} \mathrm{C}\right)}{d x}=D_{\mathrm{HC}} \frac{d(\mathrm{HC})}{d x} \\
\int^{x} R d x=-D_{\mathrm{HL}} \frac{d(\mathrm{HL})}{d x}-D_{\mathrm{L}} \frac{d(\mathrm{~L})}{d x}
\end{gathered}
$$

Equation 17 states that the rate of $\mathrm{H}_{2} \mathrm{C}$ reaching a plane at $x$ is equal to the rate of $\mathrm{HC}$ leaving the plane in an opposite direction. The right side of equation 18 gives the outward diffusion rate of total lactate at any position $x$ in the plaque. The integral in equation 18 gives the rate of acid production in the region between $\mathrm{x}$ and the enamelplaque interface $(x=0)$.

Assuming the net reaction: $\mathrm{Ca}_{10}\left(\mathrm{PO}_{4}\right)_{6}$ $(\mathrm{OH})_{2}+8 \mathrm{H}^{+}=10 \mathrm{Ca}^{2}+6 \mathrm{HPO}_{4}+$ $2 \mathrm{H}_{2} \mathrm{O}$, which defines the congruency of the reaction, the following relations at $0 \leqslant x \leqslant h$ may be obtained:

$$
\begin{gathered}
0.6 D_{\mathrm{Ca}} \frac{d(\mathrm{Ca})}{d x}=D_{\mathrm{HP}} \frac{d(\mathrm{HP})}{d x}+ \\
D_{\mathrm{H}_{2} \mathrm{P}} \frac{d\left(\mathrm{H}_{2} \mathrm{P}\right)}{d x} \\
-0.8 D_{\mathrm{Ca}} \frac{d(\mathrm{Ca})}{d x}={ }_{d}^{x} R d x+ \\
D_{\mathrm{HL}} \frac{d(\mathrm{HL})}{d x}+D_{\mathrm{H}_{2} \mathrm{C}} \frac{d\left(\mathrm{H}_{2} \mathrm{C}\right)}{d x}+ \\
D_{\mathrm{H}} \frac{d(\mathrm{H})}{d x}+D_{\mathrm{H}_{2} \mathrm{P}} \frac{d\left(\mathrm{H}_{2} \mathrm{P}\right)}{d x}
\end{gathered}
$$

Eliminating all the $\phi$ 's, equation 1 to 8 can be combined and integrated using conditions given by equations 11 to 20 . The first integration* gives the following set of equations:

$$
\begin{gathered}
G=-D_{\mathrm{Ca}} \frac{d(\mathrm{Ca})}{d x} \\
0.6 G=-D_{\mathrm{HP}} \frac{d(\mathrm{HP})}{d x}-D_{\mathrm{H}_{2} \mathrm{P}} \frac{d\left(\mathrm{H}_{2} \mathrm{P}\right)}{d x} \\
{ }_{0}{ }^{x} R d x=-D_{\mathrm{L}} \frac{d(\mathrm{~L})}{d x}-D_{\mathrm{HL}} \frac{d(\mathrm{HL})}{d x} \\
0.8 G={ }_{0}{ }^{x} R d x+D_{\mathrm{HL}} \frac{d(\mathrm{HL})}{d x}+ \\
D_{\mathrm{H}_{2} \mathrm{C}} \frac{d\left(\mathrm{H}_{2} \mathrm{C}\right)}{d x}+D_{\mathrm{H}} \frac{d(\mathrm{H})}{d x}+ \\
D_{\mathrm{H}_{2} \mathrm{P}} \frac{d\left(\mathrm{H}_{2} \mathrm{P}\right)}{d x}
\end{gathered}
$$

* Only the steady-state treatment was considered here. Based on the diffusion theory ${ }^{14}$ and experimental work, ${ }^{15}$ this assumption should be meaningful for plaque thicknesses of a few millimeters or less. Because plaque-action times are the order of hours, the steady-state attainment time, which is the order of minutes, is negligible. The linear plaque growth rates were assumed to be small compared to the steadystate attainment time. 


$$
D_{\mathrm{H}_{2} \mathrm{C}} \frac{d\left(\mathrm{H}_{2} \mathrm{C}\right)}{d x}+D_{\mathrm{HC}} \frac{d(\mathrm{HC})}{d x}=0
$$

Equation 21 defines the demineralization rate, $G$. Physically, $G$ is the negative of the rate of diffusion of $\mathrm{Ca}^{2}+$ through the plaque. Equation 22 gives the relation between the dissolution rate and the diffusion rate of total phosphate. Equation 23 relates the acid production rate to the diffusion rate of total lactate. Equation 24 relates the total acid diffusion rate to the demineralization rate. Equation 25 describes the diffusion behavior of carbonates in the plaque.

Equates 21 to 25 are integrated from the limits $x=0$ to $x=h$, and the concentrations of various species at each boundary are substituted. The following equations can be obtained:

$$
\begin{aligned}
& G h=D_{\mathrm{Ca}}\left[(\mathrm{Ca})_{o}-(\mathrm{Ca})_{h}\right] \\
& 0.6 G h=D_{\mathrm{HP}}\left[(\mathrm{HP})_{o}-(\mathrm{HP})_{h}\right]+ \\
& D_{\mathrm{H}_{2} \mathrm{P}}\left[\left(\mathrm{H}_{2} \mathrm{P}\right)_{0}-\left(\mathrm{H}_{2} \mathrm{P}\right)_{h}\right]
\end{aligned}
$$

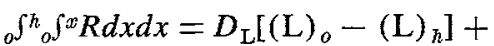

$$
\begin{aligned}
& D_{\mathrm{HL}}\left[(\mathrm{HL})_{0}-(\mathrm{HL})_{h}\right]
\end{aligned}
$$

$0.8 G h={ }_{0} \int_{0}^{h} \int^{x} R d x d x+D_{\mathrm{HL}}\left[(\mathrm{HL})_{h}-\right.$

$\left.(\mathrm{HL})_{0}\right]+D_{\mathrm{H}_{2} \mathrm{C}}\left[\left(\mathrm{H}_{2} \mathrm{C}\right)_{h}-\left(\mathrm{H}_{2} \mathrm{C}\right)_{0}\right]+$

$$
\begin{aligned}
D_{\mathrm{H}}\left[(\mathrm{H})_{h}-\right. & \left.(\mathrm{H})_{o}\right]+ \\
& D_{\mathrm{H}_{2} \mathrm{P}}\left[\left(\mathrm{H}_{2} \mathrm{P}\right)_{h}-\left(\mathrm{H}_{2} \mathrm{P}\right)_{0}\right]
\end{aligned}
$$

$$
\begin{aligned}
& \left.D_{\mathrm{H}_{2} \mathrm{C}}\left[\mathrm{H}_{2} \mathrm{C}\right)_{o}-\left(\mathrm{H}_{2} \mathrm{C}\right)_{h}\right]+ \\
& D_{\mathrm{HC}}\left[(\mathrm{HC})_{o}-(\mathrm{HC})_{h}\right]=0
\end{aligned}
$$

With the combination of equations 11 to 16 , equations 26 to 30 can be solved algebraically to give the following equations:

$$
\begin{aligned}
& { }_{0} \int_{a}^{h}{ }_{a}{ }^{x} R d x d x+D_{\mathrm{HL}}(\mathrm{HL})_{h}- \\
& \frac{{ }_{0}{ }^{h}{ }_{0}{ }^{x} R d x d x+D_{\mathrm{L}}(\mathrm{L})_{h}+D_{\mathrm{HL}}(\mathrm{HL})_{h}}{\frac{D_{\mathrm{L}} K_{\mathrm{HL}}}{(\mathrm{H})_{o}}+D_{\mathrm{HL}}}+ \\
& (\mathrm{H})_{h}-(\mathrm{H})_{o}+\left(\mathrm{H}_{2} \mathrm{P}\right)_{h}- \\
& \frac{0.6 G h+D_{\mathrm{HP}}(\mathrm{HP})_{h}+D_{\mathrm{H}_{2} \mathrm{P}}\left(\mathrm{H}_{2} \mathrm{P}\right)_{h}}{\frac{D_{\mathrm{HP}} \mathrm{K}_{2 \mathrm{P}}}{(\mathrm{H})_{o}}+D_{\mathrm{H}_{2} \mathrm{P}}}+
\end{aligned}
$$

$$
\begin{gathered}
\frac{\left(\mathrm{H}_{2} \mathrm{C}\right)_{h}-}{D_{\mathrm{H}_{2} \mathrm{C}}\left(\mathrm{H}_{2} \mathrm{C}\right)_{h}+D_{\mathrm{HC}}(\mathrm{HC})_{h}} \\
\frac{D_{\mathrm{HC}_{\mathrm{H}_{2} \mathrm{C}}} \mathrm{K}}{(\mathrm{H})_{o}}+D_{\mathrm{H}_{2} \mathrm{C}}
\end{gathered}
$$

Substitution of equation 10 into equation 9 gives the following equation at $0 \leqslant x \leqslant h$ :

$$
D_{X} \frac{d^{2}(\mathrm{~S})}{d x^{2}}=\frac{k_{1}(\mathrm{~S})}{1+k_{2}(\mathrm{~S})}
$$

This nonlinear, second order differential equation describes the glycolysis and diffusion behavior of the substrate, glucose, in the plaque. By applying the boundary conditions:

$$
\begin{aligned}
& \text { at } x=0, \\
& \qquad(S)=(S)_{0} \text { and } \frac{d(S)}{d x}=0 \text {, and }
\end{aligned}
$$$$
\text { at } x=h \text {, }
$$

$$
(\mathrm{S})=(\mathrm{S})_{h} \text {, }
$$

the following equation can be obtained by the integration of equation 33 :

$$
\begin{aligned}
& \frac{d(\mathrm{~S})}{d x}=\sqrt{\frac{2 k_{1}}{k_{2} D_{\mathrm{S}}}} \\
& {\left[(\mathrm{S})-(\mathrm{S})_{0}-\frac{1}{k_{2}} \ln \frac{1+k_{2}(\mathrm{~S})}{1+k_{2}(\mathrm{~S})_{o}}\right]^{1 / 2}}
\end{aligned}
$$

(S) is the concentration of substrate and is a function of $x,(S)_{o}$ is the value of (S) at $x=0$, and $(S)_{h}$ is the substrate level in the saliva. The boundary conditions have the physical meanings that the enamel is impermeable to the substrate, and that the saliva serves as a source of substrate for the enzymatic reaction in the plaque.

Calculations With THE Model.-Equations 31 and 32 are two equations in two unknowns, $G$ and $(\mathrm{H})_{0}$. They are solvable in principle, but do not succumb to analytical methods. Therefore, a numerical method was used for solving these equations. In order to do this, the implicit 
double integral with the integrand $R$ in equation 31 must be evaluated first.

Using Simpson's rule ${ }^{16}$ for integration and successive approximations, equation 34 can be solved numerically on the computer to give $\mathrm{S}$ as a function of $x$. A schematic profile of glucose in the plaque is shown in Figure 3. Values of $(S)$ are substituted into equation ten to obtain values of $R$ as a function of $x$. The $R$ values are then substituted into equation 31 , and with equation 32 the enamel demineralization rate, $G$, under various plaque and saliva conditions may be computed.

CONSIDERATIONS IN THE SELECTION OF CONSTANTS FOR CALCULATIONS.-The main problem in studying or applying the model with in vivo or in vitro data is the proper choice of the values for the parameters entering into equations 31 and 32 . This involves the selection of boundary conditions such as saliva composition, substrate level, and enzymatic activity of the plaque. Also a choice of suitable values for the solubility product of hydroxyapatite, the dissociation constants of the various acid species and the diffusion coefficients is necessary.

Except for the solubility product of hydroxyapatite and the dissociation constant of lactic acid, the values of the thermodynamic constants suggested by Brown ${ }^{17}$ or those from the tabulations of Robinson and Stokes ${ }^{18}$ were used in the calculations. The solubility product of hydroxyapatite was consistent with the value which has been used to explain the

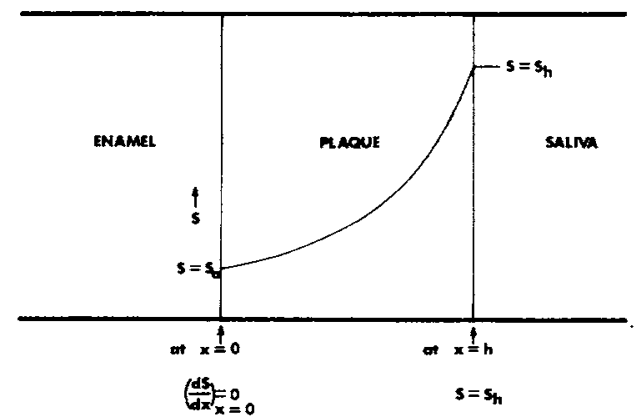

FIG 3.-A schematic plot showing the concentration profile of glucose in the plaque. This may be obtained by solving equation 33 with the boundary conditions at $x=0$ and $x=h$. dissolution behavior of block enamel, enamel powder, and synthetic apatite in weak acid buffer systems. ${ }^{1-5}$

The composition of saliva may differ among individuals. Also the composition varies with location of the mouth because of difference in flow rates and in saliva secreted from the parotid and submaxillary glands.

The first approximation in seeking out the primary effects shall be only concerned with the well-established characteristics of saliva, namely, the supersaturation with respect to hydroxyapatite and the buffer capacity. These values were taken from Schmidt-Nielsen for calcium and phosphate ions and from Shannon et al for carbonate; both values were tabulated by McCann and Brudevold. ${ }^{19}$ The saliva lactate was assumed effectively negligible when saliva flow was steady. Four values of saliva glucose levels from 0.01 to $10 \%$ were used.

The dependence of an enzyme-catalyzed reaction on substrate concentration frequently obeys the Michaelis-Menten rate equation. The kinetic data on the glycolysis reaction of Muntz ${ }^{10}$ and of Stralfors ${ }^{11}$ were fitted to this equation. Then the Lineweaver-Burk method, ${ }^{12}$ which involves the use of the reciprocal form of the equation, was used to obtain the apparent Michaelis constants, $k_{1}$ and $k_{2}$.

Plaque materials or bacterial flora from different individuals have been shown to have different activities in acid production. ${ }^{10}$ In order to cover the variabilities in acid production of the plaque, three pairs of Michaelis constants were used for the present analyses to represent high, medium, and low. The dependence of the acid production rate on substrate concentration for each of these pairs of constants is shown in Figure 4. At high substrate concentrations, the acid production rates of $A, B$, and $C$ tended to level off, and showed the high, medium, and low in capacity, respectively. According to these results for both $A$ and $C$, the enzymatic pathways of the bacteria are saturated with substrate more quickly than that of $B$. For calculations involving plaques $A, B$, and $C$, a value of $D=1 \times 10^{-5} \mathrm{~cm}^{2} / \mathrm{sec}$ was used for the diffusion coefficients of all the species involved, including glucose. As a further examination of the model for these cases, $D_{g}=1 \times 10^{-5} \mathrm{~cm}^{2} / \mathrm{sec}$ was used for the 


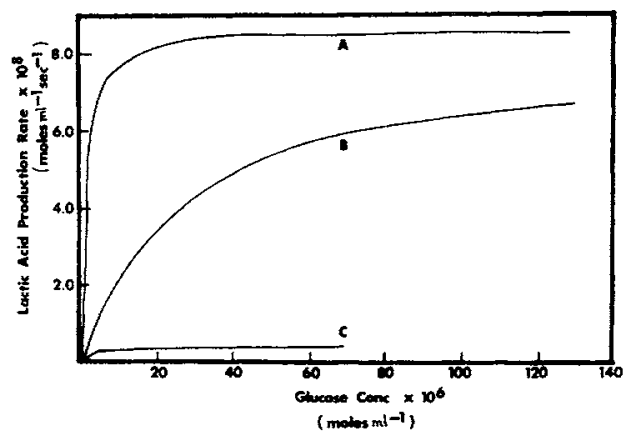

FIG 4.--Acid production rate vs substrate concentration for plaques $A, B$, and $C$.

diffusion coefficient of glucose and $D_{0}=$ $1 \times 10^{-6} \mathrm{~cm}^{2} / \mathrm{sec}$ was used for all of the other species involved. Also, $D=1 \times 10^{-6}$ $\mathrm{cm}^{2} / \mathrm{sec}$ for all the species, including glucose, was used for calculations involving plaque $C$.

Values of all the parameters entering the model, chosen independently, are listed in Table 1. These data then permitted the calculations for the rate of demineralization of enamel as a function of plaque thickness for the different glucose levels in the saliva and enzymatic activities of the plaque. Enzymatic activity of the plaque, plaque thickness, and saliva substrate level were allowed variations of the order of magnitude expected for in vivo situations.

CONSIDERATIONS IN THE IN VIVO EXPERI-

TABLE 1

Selected Values of Parameters Used in Calculations with the Model

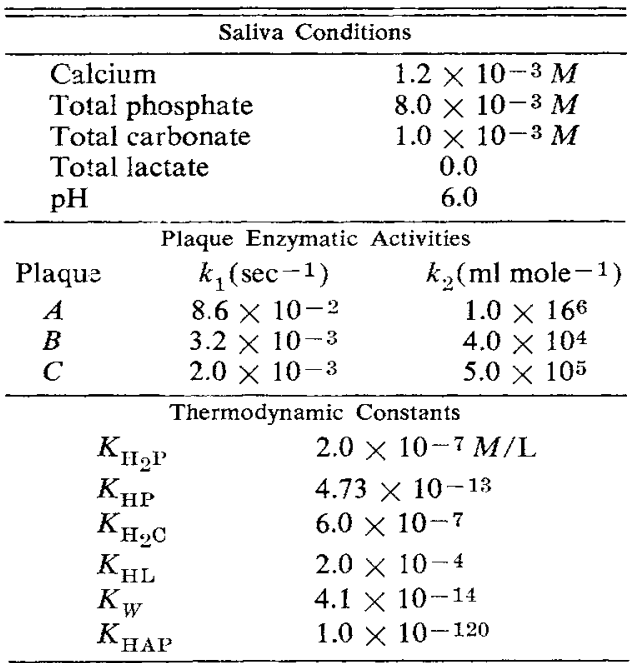

MENTS.-In an effort to investigate the predictions of the model, correlations between the model and in vivo experimental observations were attempted. These experiments were conducted with a modification of a design that used enamel slabs intraorally in four human volunteers. ${ }^{20,21}$ In short, enamel slabs were mounted in prosthetic devices with one or more layers of Teflon* (0.37) or Dacron* (0.15) gauze covering in order to accelerate bacterial colonization. The thickness of the covering material is a strong factor that limits bacterial growth. Presumably the gauze functions similarly to the pits and fissures of teeth in natural caries, ie, causing conditions of various degrees of decrease in enamel microhardness. Similar microhardness change is obtained in vitro on exposure of enamel slabs to acidic buffers. Although the microhardness measurement is an indirect technic for estimating demineralization, it is extremely sensitive to partial enamel dissolution as undoubtedly is the case in incipient caries. The relationship between the incipient calcium and phosphate loss from enamel and the microhardness decrease is surprisingly quantitative when the conditions of demineralization are carefully controlled. Although bacterial colonization and enzymatic activity in the human mouth is far from the controlled conditions of an in vitro system, in terms of similarity to natural caries, the in vivo approach provides information inaccessible to other experimental designs. Undoubtedly the growth and enzymatic activity of the bacteria in their natural habitat would be different than in any other environment.

Considering the aforementioned physical model, two degrees of freedom in causing enamel changes are still left, ie, the enzymatic activity and the saliva substrate level. Since data for both of these variables were not available from the testing subjects, the exact in vivo experimental relationships among enzymatic activity, saliva substrate level, and enamel reactions were undetermined.

\section{Results}

Figure 5 gives the results of calculations for the demineralization rate in the special case for which a constant acid

* United States Catheter and Instrument Corporation, Glenn Falls, NY. 


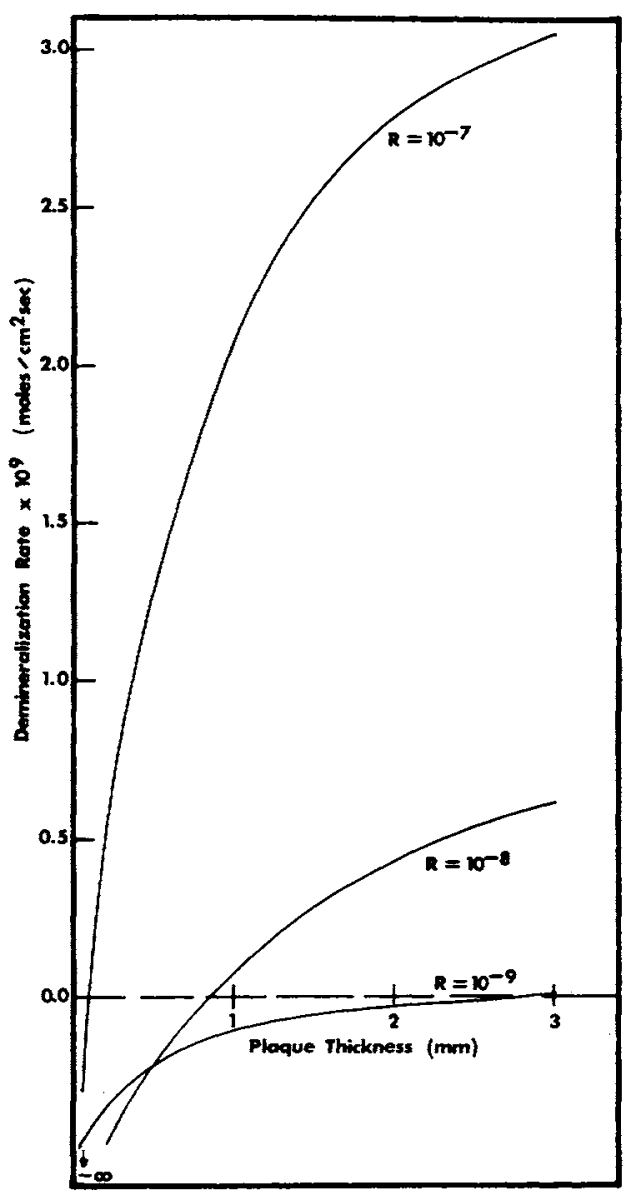

FIG 5.-Effects of the plaque thickness on the enamel demineralization for three constant acid production rates, $R$, in the plaque.

production rate, $R$, was assumed throughout the plaque. This situation corresponds to the situation in which the substrate is "swamping" and, therefore, eliminates the importance of the diffusion rate of substrate in the plaque. The three curves correspond to three acid production rates that are of the order of magnitude encountered in real bacterial plaques and are also comparable to those shown in Figure 4. For each $R$, there is a critical plaque thickness for the transition from remineralization (negative values of $G$ ) to demineralization. This critical plaque thickness is given by the crossing point on the abscissa, $h$. When a plaque was of a thickness less than the critical value, with saliva action overwhelming the plaque action, remineralization was supported at the enamel-plaque interface. For plaques of thicknesses greater than the critical value, the plaque action dominated and demineralization was favored. As clearly shown, a smaller $R$ gave a larger plaque thickness. This is so because the saliva action can tolerate a larger barrier in counteracting a smaller plaque action. On the other hand, a larger $R$ and a thicker plaque may greatly overwhelm the saliva action and cause a high demineralization rate.

Figure 6 gives the results of calculations, showing the glucose concentration profiles for the general situation involving enamel demineralization with simultaneous substrate (glucose) diffusion and acid production in the plaque. For these calculations the conditions of plaque $A$ were used with thicknesses of $0.1,0.5,1.0,2.0$, and 3.0 $\mathrm{mm}$ at four saliva glucose levels, viz, $(S)=10,1,0.1$, and $0.01 \%$. For convenience, the logarithms of the substrate concentrations in the plaque have been plotted.

For $(S)=10 \%$, the concentration profiles (Fig 6), show that the enzymatic pathways throughout the plaque were essentially saturated with substrate for all thicknesses. This is suggested by the plot for plaque $A$ in Figure 4. At such saliva substrate levels, the plaque action of plaque $A$ was mainly controlled by its enzymatic activity. When the saliva substrate level was low, eg, (S) $=0.01 \%$, its concentration dropped rapidly from the plaquesaliva interface. For thicker plaque, ( 2 to 3 $\mathrm{mm}$ ), this was especially evident. In these instances, the substrate was unable to reach the enamel-plaque boundary, and most of the inner plaque was considerably undersaturated with respect to the substrate. Because of the lack of substrate, the acid

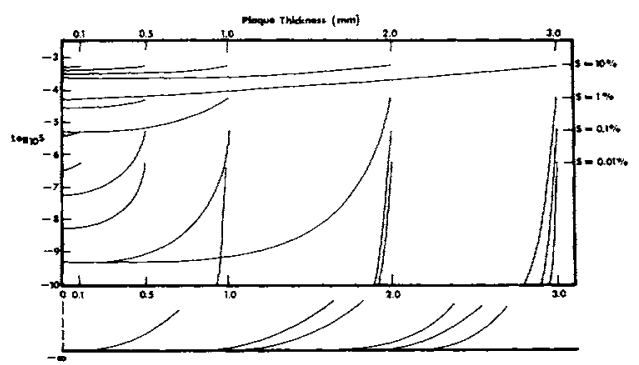

FIG 6.- Glucose profiles in plaque $A$ (with $k_{1 \mathrm{~A}}, k_{2 \mathrm{~A}}$ ) shown for five plaque thicknesses from 0.1 to $3.0 \mathrm{~mm}$. 
was not produced at a rate comparable to the maximum production possible for the enzyme systems; the acid production rate principally depended on the rate of substrate being supplied.

At the intermediate substrate levels, ie, for $(S)=0.1$ to $1.0 \%$, the relationship between the plaque action, the enzymatic activity, and the diffusion of substrate was complicated by the plaque thickness effect. A thick plaque, $(h=2$ to $3 \mathrm{~mm})$, presented a greater barrier to the diffusion of the substrate, and hence limited the substrate to the outer region of the plaque and left most of the inner portion in an undersaturated state. The overall effect was to make the plaque action more dependent on the diffusion of substrate than on the enzymatic activity. A thinner plaque, $(h=$ 0.5 to $1.0 \mathrm{~mm}$ ), allowed more substrate to reach the enamel-plaque interface; the plaque action was more dependent on the enzymatic activity than on the diffusion rate of the substrate.

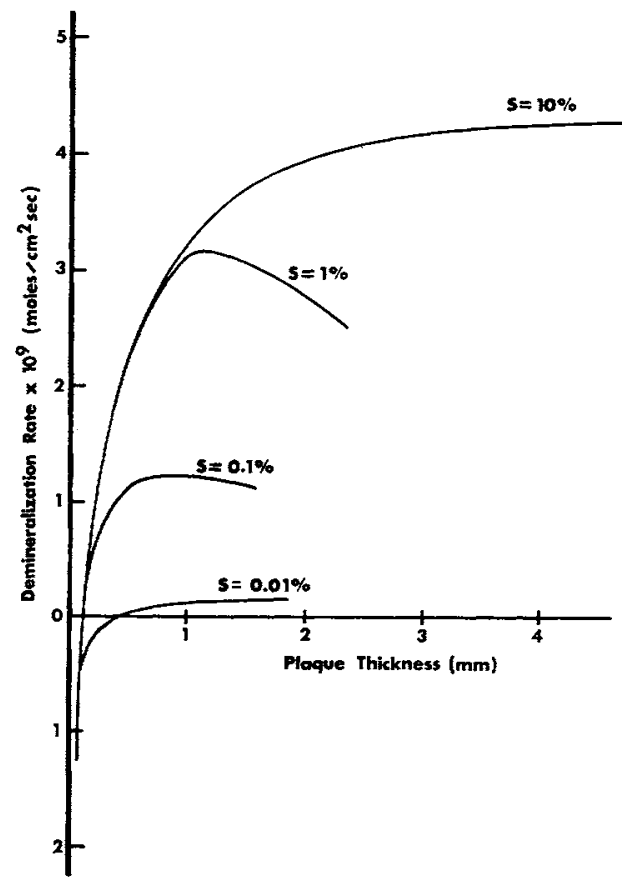

FIG 7.--Effect of the plaque thickness on the enamel demineralization in plaque $A$ shown for four saliva glucose levels, $S$, with diffusion coefficient $D=1.0 \times 10^{-5} \mathrm{~cm}^{2} \mathrm{sec}^{-1}$ for all species.
The results of the calculations carried out for the demineralization rate as a function of plaque thickness for plaques $A, B$, and $C$ at four saliva substrate levels are shown in Figures 7-9. The curves all show the same general features as those in Figure

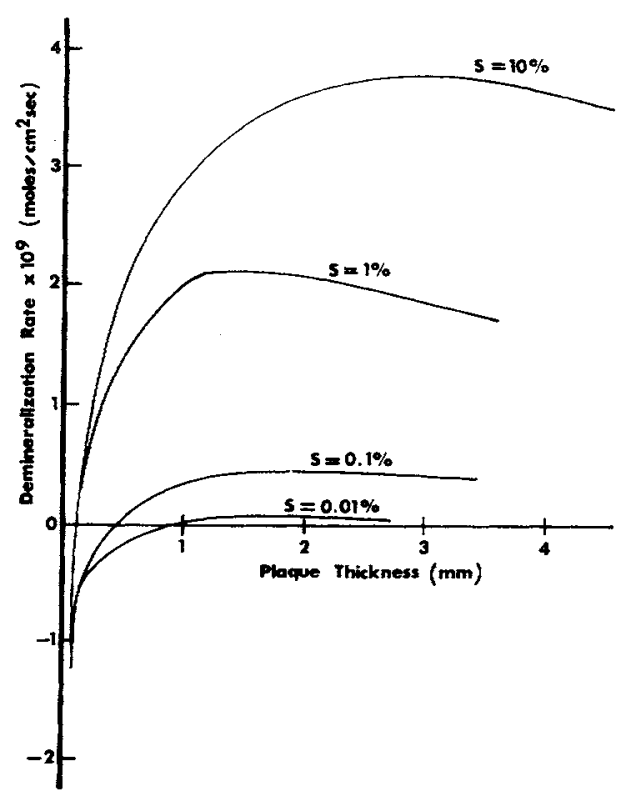

FIG 8.-Effect of the plaque thickness on the enamel demineralization in plaque $B$ shown for four saliva glucose levels, S, with $D=$ $1.0 \times 10^{-5} \mathrm{~cm}^{2} \mathrm{sec}^{-1}$ for all species.

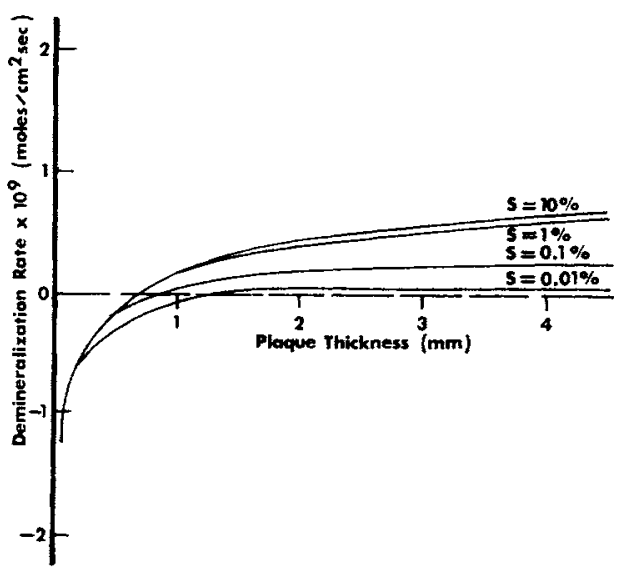

Fig 9.-Effect of the plaque thickness on the enamel demineralization in plaque $C$ shown for four saliva glucose levels, $S$, with $D=1.0 \times$ $10^{-5} \mathrm{~cm}^{2} \mathrm{sec}^{-1}$ for all species. 
5. The demineralization behavior now involved, however, the consideration of the diffusion of substrate in the plaque.

In Figure 7 (for plaque $A$ ) when $(\mathrm{S}$ ) $=$ $10 \%$, as discussed previously, the enzymatic pathways were saturated with substrate and the plaque action was independent of the diffusion rate of substrate. A demineralization behavior similar to that shown in Figure 5 was therefore expected. When $\mathbf{S}=1 \%$ for thin plaques up to $h=$ $0.8 \mathrm{~mm}$, demineralization rates essentially the same as those for $(S)=10 \%$ were obtained, and the enzymatic pathways were saturated throughout the plaque for both (S) $=1 \%$ and $(\mathrm{S})=10 \%$.

When $(S)=1 \%$, the dependence of the enamel demineralization rate on plaque thickness for thicker plaques was complicated by having a maximum value at $h=$ $1 \mathrm{~mm}$. From Figure 6 for $h=2.0 \mathrm{~mm}$, the following substrate levels at the enamelplaque interface and the plaque-saliva interface can be obtained: $(\mathrm{S})_{0}=5.55 \times$ $10^{-10} \mathrm{moles} / \mathrm{ml}$ and $(\mathrm{S})_{h}=5.55 \times 10^{-5}$ moles $/ \mathrm{ml}$ respectively. By using equation ten and the Michaelis constants for plaque $A$, the acid production rates at the two interfaces can be calculated as $(R)_{o}=$ $4.7 \times 10^{-11}$ moles $/ \mathrm{ml} \mathrm{sec}$ and $(R)_{h}=$ $8.6 \times 10^{-8}$ moles $/ \mathrm{ml}$ sec respectively. Figure 4 for plaque $A$ clearly shows that $(R)_{h}$ was at the plateau region where acid was produced at full capacity for the enzyme systems. On the other hand, $(R)_{0}$ shows that the rate was limited by the deficiency of substrate, as shown by the concentration profiles in Figure 6. Apparently $(R)_{0}$ was negligible compared to $(R)_{h}$. Acid was produced at the outer part of the plaque, but because of the deficiency of substrate, the acid was produced only at a very low rate. A lesser amount of acid thus reached the enamel-plaque interface, and effected a lower demineralization rate than when the plaque thickness was around $1.0 \mathrm{~mm}$. However, for $h=1.0 \mathrm{~mm}$, with $(R)_{0}=7.28 \times 10^{-8} \mathrm{~mole} / \mathrm{ml} \mathrm{sec}$, which is close to $(R)_{h}$, much of the inner plaque can produce acid close to full capacity. Thus a more than sufficient amount of acid was produced in overcoming the increase in the saliva action caused by a thinner barrier; more acid reached the enamelplaque interface, which caused a higher demineralization rate.
For $(S)=0.1 \%$ demineralization behavior was similar to that for $(S)=1 \%$, except that the maximum in demineralization rate became less distinct. This plot showed that at lower saliva substrate level, where plaque action was lower, the effect of saliva action on the demineralization rate became more significant. The saliva action tended to level off the demineralization rate with respect to plaque thickness. This was distinctly shown for $(S)=0.01 \%$.

Figure 8 presents the results with plaque $B$. The demineralization behavior here was generally similar to that with plaque $A$. When the substrate level was high, the enzyme systems of plaque $B$ approached the maximum capacity of plaque $A$ in acid production. As can be calculated from equation 10 , at $(S)=10 \%$ plaque $B$ gave an acid production rate of $R_{B}=7.65 \times$ $10^{-8} \mathrm{moles} / \mathrm{ml} \mathrm{sec}$. For plaque $A$, a value of $R_{A}=8.60 \times 10^{-8} \mathrm{moles} / \mathrm{ml}$ sec was obtained, which shows the closeness in plaque actions for plaque $A$ and $B$ at (S) $=$ $10 \%$. Therefore, comparable demineralization rates were observed for the two plaques. However, at lower substrate levels, the difference in plaque actions of the two plaques became greater. For example, at $\mathrm{S}=1 \% R_{B}=5.51 \times 10^{-8} \mathrm{moles} / \mathrm{ml} \mathrm{sec}$, and $R_{A}=8.60 \times 10^{-8} \mathrm{moles} / \mathrm{ml} \mathrm{sec}$.

A larger difference in demineralization rates between $\mathrm{S}=10 \%$ and $\mathrm{S}=1 \%$ was observed for plaque $B$ than for plaque $A$. For this reason, the identical demineralization behavior observed in plaque $A$ for $h<$ $0.8 \mathrm{~mm}$ at $\mathrm{S}=1$ and $10 \%$ was absent in plaque $B$. Similarly, lower demineralization rates were observed for $\mathrm{S}=0.1$ and $0.01 \%$ in plaque $B$ than in plaque $A$.

The enzymatic pathways of plaque $C$ were easily saturated with substrate and gave low capacity in acid production. Because the plaque action was low, the demineralization rates in plaque $C$, as presented in Figure 9, were generally lower than were those of plaques $A$ and $B$.

The results of using $D_{g}=1 \times 10^{-5}$ $\mathrm{cm}^{2} / \mathrm{sec}$ and $D_{0}=1 \times 10^{-6} \mathrm{~cm}^{2} / \mathrm{sec}$ are shown in Figures $10-12$. In relation to the previous discussion, the smaller $D_{0}$ made the diffusion flux of all the species except glucose smaller. Thus the glucose diffusion rates in the plaque were unchanged, and the acid production rates also remained constant. However, the acid species $\mathrm{H}^{+}$, 


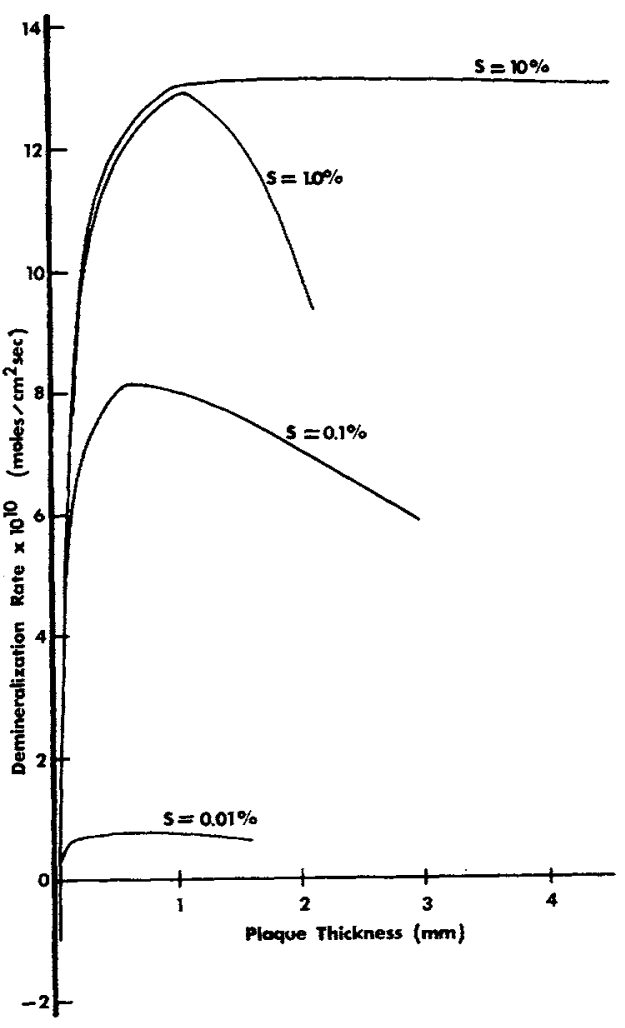

FIG 10.-Effect of the plaque thickness on the enamel demineralization rate in plaque $A$ shown for $D_{g}=1.0 \times 10^{-5} \mathrm{~cm}^{2} \mathrm{sec}^{-1}$ and $D_{o}=1.0 \times 10^{-6} \mathrm{~cm}^{2} \mathrm{sec}^{-1}$.

$\mathrm{HL}$, and $\mathrm{H}_{2} \mathrm{CO}_{3}$ approached the enamelplaque interface with a smaller rate. Also, the species disengaged from the mineral phase, ie, the calcium and the phosphates, which had smaller diffusion fluxes outward, tended to build up their concentrations at the interface. This lowering in acid diffusion rate to the enamel-plaque interface, and the local accumulations of common ions at the interface, caused a general lowering in the demineralization rates. The saliva action was also reduced because of the decrease in the diffusion rates of the species moving from the saliva toward the enamel-plaque interface. The effect of this was reflected in the thinner critical plaque thicknesses as compared to those in Figures 7-9.

The results of using $D=1 \times 10^{-6} \mathrm{~cm}^{2} /$ sec for all the species for plaque $C$ are

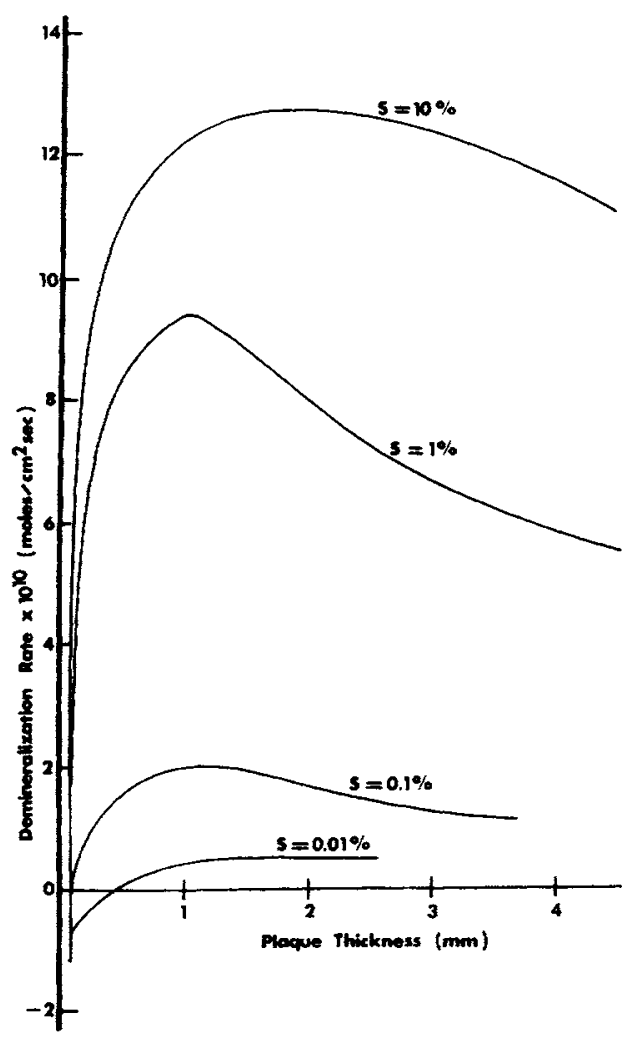

FIG 11.-Effect of the plaque thickness on the enamel demineralization in plaque $B$ with $D_{g}=1.0 \times 10^{-5} \mathrm{~cm}^{2} \mathrm{sec}^{-1}$ and $D_{0}=1.0 \times$ $10-6 \mathrm{~cm}^{2} \mathrm{sec}^{-1}$.

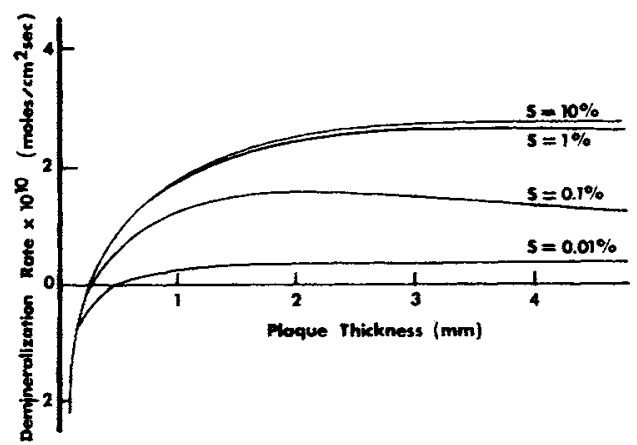

FIG 12.-Effect of the plaque thickness on the enamel demineralization in plaque $C$ with $D_{g}=1.0 \times 10^{-5} \mathrm{~cm}^{2}$ second -1 and $D_{o}=1.0$ $\times 10^{-6} \mathrm{~cm}^{2} \mathrm{sec}^{-1}$. 


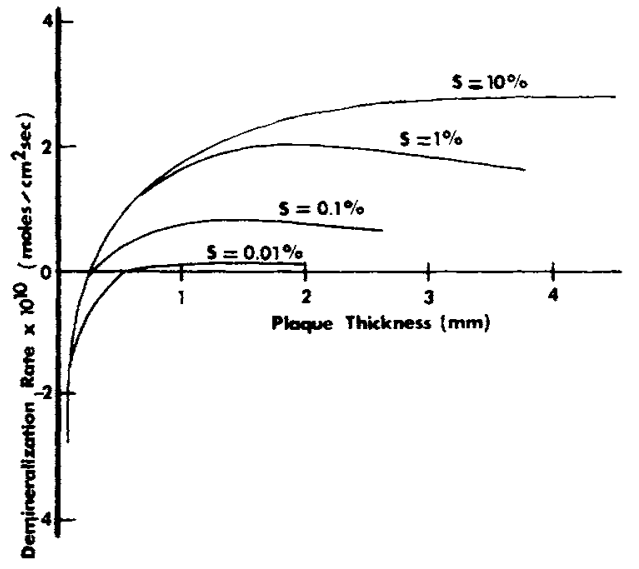

FIG 13.-Effect of the plaque thickness on the enamel demineralization in plaque $C$ with $\mathrm{D}=1.0 \times 10^{-6} \mathrm{~cm}^{2} \mathrm{sec}^{-1}$.

shown in Figure 13. Acid production rates decreased because of the reduction in the diffusion rate of glucose. In comparison to Figure 12, Figure 13 shows that at high substrate levels, eg, $\mathrm{S}=10 \%$, the demineralization behavior was essentially unchanged and the enzymatic pathways were saturated throughout the plaque, regardless of the reduction in the rate of substrate transport. A maximum shown for $\mathrm{S}=1 \%$ in Figure 13 was caused by the lowering in plaque action for thicker plaques and the smaller diffusion rate of the substrate.

\section{Discussion}

General CONSIDERATIONS OF THE MODEL.-The ionic equilibriums at the enamel-plaque interface were described by the following processes. In the plaque, lactic acid (HL) is steadily produced by the bacteria at a rate governed by both the enzymatic activity of the bacteria and glucose concentrations. Because of this, the acid ions and molecules tend to diffuse toward both the enamel-plaque interface and the plaque-saliva interface. They interact, in the case of demineralization, with ions $\mathrm{H}_{2} \mathrm{PO}-4, \mathrm{HPO}_{4}, \mathrm{HCO}_{3}^{-}$, and lactate (L) diffusing outward from the enamelplaque interface, and with $\mathrm{HCO}_{3}^{-}$diffusing inward from the plaque-saliva interface. In remineralization, they interact with $\mathrm{H}_{2} \mathrm{PO}_{4}^{-}, \mathrm{HPO}_{4}^{-}$, and $\mathrm{HCO}_{3}^{-}$diffusing in- ward from the saliva and with $\mathrm{HC}_{3}^{-}$and lactate diffusing outward. The reactions are limited by the dissociation constants for $\mathrm{H}_{2} \mathrm{PO}-\mathrm{H}_{2} \mathrm{CO}_{3}$, and $\mathrm{HL}$. The demineralization or remineralization, which is a result of the equilibrium at the enamelplaque interface, is determined by the saliva conditions and by the congruent reaction between the acid species that reach the enamel-plaque interface and hydroxyapatite. The saliva operates as a sink for ions and molecules diffusing out of plaque, as well as a source of bicarbonate and glucose for acid production in the plaque. In demineralization, it is also a sink for the products of enamel decalcification; in remineralization, it is a source of calcium and phosphates. Under certain conditions, the plaque acts as a pump to decalcify the mineral against a net thermodynamic activity gradient. Thus, decalcification and the subsequent transport of the products into the saliva sink may take place even though the saliva is supersaturated with respect to apatite.

Thus the resultant roles played by the plaque and the saliva in the enamel-plaquesaliva system can be considered in terms of plaque action and saliva action. Plaque action, which is physically well-defined in the model, is the process involving the enzymatic activity of the plaque, the diffusion of substrate in the plaque, and plaque thickness. As the result of a strong plaque action, a sufficient amount of acid species may reach the enamel-plaque interface and cause enamel demineralization. Saliva action is the process involving plaque thickness, buffer capacity, and the supersaturated state of the saliva. Saliva action may overwhelm a weak plaque action and thus, prevent enamel demineralization and support enamel remineralization. The saliva action is especially significant when the plaque is thin if the enzymatic activity is low.

Correlation of the Results of THE MODEL WITH CLINICAL SITUATIONS.-If a carious lesion is initiated by the dissolution of the mineral phase of the enamel, then the demineralization rate, $G$, as defined previously, should represent the tendency for caries development. In considering the tendency for caries formation, 
the immediate variables are the enamel conditions, the enzymatic activity of the plaque in acid production, the saliva substrate level for acid production, the plaque thickness, and the saliva conditions.

Because the enamel was assumed to be hydroxyapatite and the saliva conditions were kept constant by choosing one set of data (Table 1 ), only three variables represent the three degrees of freedom. When any two variables are kept constant, the relation between enamel reactions and the third variable may be found. For example, tooth enamel that is covered with $1.0 \mathrm{~mm}$ thick plaque has enzymatic activity corresponding to that of plaque $A$, and is bathed in saliva containing $0.1 \%$ glucose, is predicted by the model (Fig 7) to be demineralized at a rate of $1.2 \times 10^{-9}$ moles $/ \mathrm{ml}^{-1} \mathrm{sec}^{-1}$. Thus an enamel specimen should be softened to a certain degree in a specific period of time under these conditions. On the other hand, for a plaque of thickness $0.1 \mathrm{~mm}$, with the other conditions the same, the enamel is predicted to be remineralized or rehardened to a certain degree. These results are in agreement with the observation that caries is frequently observed in the molars where deep grooves allow the formation and retention of thick plaques. These results are also consistent with calculus formation on the lingual surfaces of the lower incisors where only very thin plaque can be retained.

A comparison of enamel slab microhardness experiments with the predictions of the model is of interest because of the direct relationship between the microhardness change (KHN) and enamel demineralization. The experimental results showing the effect of plaque thickness are shown in Tables 2 and 3. For each subject enamel changes (softening or rehardening) differed to some extent when measured at different dates (particularly subjects one and two). Based on the model, these differences might be attributed to the changes in the enzymatic activity, substrate level, saliva conditions, or a combination of these in the test subjects during the testing period. Despite these variations, certain consistent observations may be noted. In Table 2, microhardness changes for plaques of thicknesses of 0.37 and $0.74 \mathrm{~mm}$ are compared. These plaque thickness effects may be correlated with the model calculations

TABLE 2

Microhardness Changes (KHN) of ENAMEl Showing the EfFect of the Thickness of Bacterial Sheltering with Teflon Gauze

\begin{tabular}{|c|c|c|c|c|c|c|c|c|}
\hline \multicolumn{3}{|c|}{ Subject 1} & \multicolumn{3}{|c|}{ Subject 2} & \multicolumn{3}{|c|}{ Subject 3} \\
\hline Date & $I^{*}$ & II & Date & $I^{*}$ & $\mathrm{II} \dagger$ & Date & $I^{*}$ & $\mathrm{II} f$ \\
\hline $4 / 9$ & -133 & -103 & $4 / 9$ & +4 & -5 & $4 / 8$ & -10 & -18 \\
\hline $4 / 22$ & -35 & -26 & $4 / 22$ & -112 & -102 & $4 / 22$ & -46 & -66 \\
\hline $5 / 13$ & -4 & -1 & $5 / 24$ & -7 & +15 & $5 / 13$ & -23 & -52 \\
\hline$\ldots$ & $\ldots$ & $\cdots$ & . & $\ldots$ & $\cdots$ & $5 / 24$ & +7 & -63 \\
\hline
\end{tabular}

* I, one Iayer of Teflon gauze $(0.37 \mathrm{~mm})$.

II, two layers of Teflon gauze $(0.74 \mathrm{~mm})$.

TABLE 3

Microhardness Changes (KHN) of Enamel Showing the Effect of the Thickness of Bacterial Sheltering with Dacron Gauze

\begin{tabular}{|c|c|c|c|c|c|c|c|c|}
\hline \multicolumn{3}{|c|}{ Subject 1} & \multicolumn{3}{|c|}{ Subject 2} & \multicolumn{3}{|c|}{ Subject 4} \\
\hline Date & $I^{*}$ & II & Date & $\mathrm{I}^{*}$ & II & $\overline{\text { Date }}$ & $0+$ & $\mathrm{II}_{\dagger}^{\dagger}$ \\
\hline $3 / 27$ & +13 & -20 & $5 / 1$ & +13 & +20 & $12 / 6$ & -16 & -23 \\
\hline $4 / 14$ & 0 & -143 & $5 / 11$ & +12 & +19 & $1 / 9$ & +28 & -36 \\
\hline $5 / 1$ & -17 & -22 & $5 / 18$ & +35 & -10 & $1 / 18$ & -6 & -3 \\
\hline $5 / 9$ & +9 & -16 & $5 / 29$ & +9 & +44 & $2 / 5$ & +22 & +30 \\
\hline $5 / 17$ & -12 & +26 & $6 / 7$ & +42 & +15 & $\ldots$ & $\ldots$ & $\ldots$ \\
\hline $5 / 25$ & -8 & $\begin{array}{r}-11 \\
-11\end{array}$ & $6 / 22$ & +13 & +26 & $\cdots$ & $\cdots$ & $\cdots$ \\
\hline $6 / 7$ & -17 & -22 & $7 / 3$ & +29 & +31 & $\ldots$ & 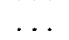 & . \\
\hline
\end{tabular}

* I, one layer of Dacron gauze $(0.15 \mathrm{~mm})$.

$\dagger$ II, two layers of Dacron gauze $(0.30 \mathrm{~mm})$.

* 0 , zero layers of Dacron gauze. 
at $(\mathrm{S})=10,1$ and $0.1 \%$ in plaque $A$ (Fig 7), and at $\mathrm{S}=10$ and $1 \%$ in plaque $B$ (Fig 8). Subject one appeared to show softening but did not show an increase in softening with plaque thickness. This was consistent with the predictions of the model in demineralization for thick plaques when demineralization rates level off with plaque thickness, eg, $\mathrm{S}=0.1 \%$ (Fig 7) and at $S=0.01 \%$ (Fig 10). Subject two also showed little dependency on plaque thickness. Subject three showed a consistent plaque thickness effect. In each experiment with this subject, the thicker plaque always gave a greater softening.

In Table 3, enamel changes with plaque of thicknesses 0.15 and $0.30 \mathrm{~mm}$ are shown. Subject one appeared to show softening with increased plaque thickness. This effect appeared to be absent at higher plaque thicknesses. Subject four showed an insignificant plaque thickness effect on softening. Subject two showed consistent rehardening of the enamel. In the physical model calculations, remineralization (Table 2) was found for plaque of thickness of $0.3 \mathrm{~mm}$ at $\mathrm{S}=0.01 \%$ (Fig 7), at $\mathrm{S}=$ 0.01 to $0.1 \%$ (Fig 8), and for any substrate level in plaque $C$ (Fig 9).

These correlations of the physical model with the microhardness experiments are preliminary. However, these findings do demonstrate the potential usefulness of the physical model approach in these situations. Ideally, other kinds of both in vitro and in vivo experiments are needed that involve the plaque-tooth-saliva system.

Future work will involve extending this model to accommodate more difficult situations. The possible complexes of calcium and phosphates in the saliva and in the plaque may be generally grouped into diffusible and nondiffusible complexes. Whereas nondiffusible complexes must be considered in nonsteady-state analyses, only diffusible complexes are important in the steady-state treatment of the model, and their effects on enamel reactions can be evaluated by redefining the demineralization rate to include the transport of the complexes. Where the enamel surface phase formation of fluorapatite or calcium fluoride is suspected, eg, as a result of topical fiuoride treatment, the appropriate boundary conditions must be used with the differential equations. The formation of extra- cellular polysaccharides from sucrose, the utilization of polysaccharides in producing acids, the accumulation of phosphates by streptococci, 22 and many other factors can be interrelated with enamel reactions in the framework of this model as more kinetic data become available. Along with these, the role of fluoride in inhibiting polysaccharide synthesis by the bacteria and the formation of ammonia in the plaque ${ }^{23}$ can be treated.

\section{Conclusions}

A dynamic model for a tooth-plaquesaliva system has been presented. It relates enamel demineralization and enamel remineralization to the production of lactic acid in the plaque from glucose.

Relationships were obtained on the basis of the consideration of simultaneous diffusion and chemical reactions of the various molecular and ionic species involved in plaque. It permitted the interrelating of important variables involved in dental caries.

The results of this model were correlated under various conditions with in vivo data. The predictions of the model for plaque thickness effects have been found to be consistent with the experimental enamel softening and rehardening observations to an order of magnitude. With refinements of the model and quantitative experimentation, cariogenicity will probably be defined more accurately, and therefore systematic progress will be made in the understanding of dental caries.

The authors appreciate the suggestions of $R$. S Manly in the early stages of this work.

\section{References}

1. Higuchi, W.I.; Gray, J.A.; HefFERrEN, J.J.; and PATEL, P.R.: Mechanisms of Enamel Dissolution in Acid Buffers, $J$ Dent Res 44:330-41, 1965.

2. Patel, P.R.: Studies on Acid Demineralization Kinetics of Enamel, PhD thesis, University of Michigan, 1965.

3. MIR, N.A.: The Mechanism of Action of Solution Fluoride upon the Demineralization Rate of Enamel, PhD thesis, University of Michigan, 1967.

4. Nelson, K.G.: Studies on the Rate of Formation of Calcium Fluoride on $\mathrm{Hy}-$ droxyapatite in Buffered Fluoride Solutions, $\mathrm{PhD}$ thesis, University of Michigan, 1968.

5. Higuchi, W.I.; Mrr, N.A.; Patel, P.R.; BECKER, J.W.; and HefFERREN, J.J.: Quan- 
titation of Enamel Demineralization Mechanisms: III. A Critical Examination of the Hydroxyapatite Model, J Dent Res 48:396409, 1969.

6. Bibby, B.G.; Gustafson, G.; and Davies, G.N.: A Critique of Three Theories of Caries Attack, Int Dent J 8:685-695, 1958.

7. Jenkins, G.N.: The Physiology of the Mouth, 3rd ed, Philadelphia: F.A. Davis Company, 1966, pp 391-400.

8. Koulourides, T., and HiguchI, W.I.: Physico-Chemical Model for Tooth Demineralization Action of Dental Plaque, $J$ Dent Res 45:1840, 1966.

9. Young, F.D.; HiguchI, W.I.; and KouLoURIDEs, T.: Physico-Chemical Model for the Dental Plaque, J Dent Res 46:1549, 1967.

10. Muntz, J.A.: Production of Acids from Glucose by Dental Plaque Material, $J$ Biol Chem 148:225-236, 1943.

11. Stralfors, A.: Investigation into the Bacterial Chemistry of Dental Plaques, Odont $T$ 58:151-341, 1950 .

12. LAIDLER, K.J.: The Chemical Kinetics of Enzyme Action, Oxford: Clarendon Press, 1958 , chap 3.

13. Olander, D.R.: Simultaneous Mass Transfer and Equilibrium Chemical Reactions, A I Ch E J 6:233-239, 1960.

14. Jost, W.: Diffusion in Solids, Liquids, Gases, New York: Academic Press Inc., Publishers, 1960, p 44.

15. Higuchi, W.I.; Parrott, E.L.; Wurster, D.E.; and HIGUCHI, T.: Investigation of Drug Release from Solids: II. Theoretical and Experimental Study of Influences of
Bases and Buffers on Rates of Dissolution of Acidic Solids, $J$ Amer Pharm Ass 47: 376-383, 1958.

16. Kaplan, W.: Advanced Calculus, Reading, Mass: Addison-Wesley Publishing Company, Inc., 1959, pp 171-172.

17. Brown, W.E.: Behavior of Slightly Soluble Calcium Phosphates as Revealed by PhaseEquilibrium Calculations, Soil Sci 90:51-57, 1960.

18. Robinson, R.A., and Stokes, R.H.: Electrolyte Solutions, London: Academic Press, Inc., 1959, p 482.

19. McCann, H.G., and Brudevold, F.: "The Mechanism of the Caries-Inhibiting Effects of Fluoride," in KRESHOVER, S.J., and MCCluRE, F.J., Environmental Variables, Washington, DC: American Association for the Advancement of Science, 1966.

20. Koulourides, T.: Dynamics of Tooth Surface-Oral Fluid Equilibrium, Advances Oral Biol 2:149-171, 1966.

21. Koulourides, T.; LASTRA, J.; Higuchi, W.I.; and Young, F.D.: Experimental Cariogenicity in the Human Mouth: Bacterial Plaque Thickness, J Dent Res 47: 1254, 1968.

22. TANzer, J.M.; Krichevsky, M.I.; and KEYES, P.H.: The Coupling of Phosphate Accumulation to Acid Production by Nongrowing Streptococci, $J$ Gen Microbiol 55: 351-360, 1969.

23. Singer, D.L., and Kleinberg, I.: $\mathrm{NH}_{3}$ Formation in Plaque In Situ, abstracted, IADR Program and Abstracts of Papers, No. $637,1969$. 